ISSN 0103-5150

Fisioter. Mov., Curitiba, v. 26, n. 2, p. página 349-360, abr./jun. 2013

Licenciado sob uma Licença Creative Commons

\title{
Capacidade funcional de idosos com osteoartrite submetidos a fisioterapia aquática e terrestre
}

\author{
Functional capacity of elderly with osteoarthritis who \\ undergone to aquatic and land physical therapy
}

\author{
Glauber de Oliveira Barduzzi ${ }^{[a]}$, Paulo Roberto Rocha Júnior ${ }^{[b]}$, \\ José Carlos de Souza Neto ${ }^{[\mathrm{c}]}$, Mariana Chaves Aveiro ${ }^{[\mathrm{d}]}$ \\ [a] Fisioterapeuta graduado na Universidade Paulista (Unip), Assis, SP - Brasil, e-mail: fisioglauber@uol.com.br \\ [b] Fisioterapeuta, professor do curso de Fisioterapia da Universidade Paulista (Unip), professor do curso de Fisioterapia e \\ Gerontologia das Faculdades Adamantinenses Integradas (FAI), mestre em Saúde Coletiva pela Universidade Estadual \\ Paulista (Unesp), Botucatu, SP - Brasil, e-mail: prochajr@terra.com.br \\ [c] Treinador esportivo, educador físico graduado no Instituto Educacional de Assis (Ieda), especialista em Fisiologia Geral e \\ do Exercício pelo Instituto Educacional de Assis (Ieda) Assis, SP - Brasil, e-mail: jcarlos_neto@hotmail.com \\ [d] Fisioterapeuta, professora adjunta do curso de Fisioterapia da Universidade Paulista (Unip), doutora em Fisioterapia pela \\ Universidade Federal de São Carlos (UFSCar), São Carlos, SP- Brasil, e-mail: mariaveiro@yahoo.com
}

\section{Resumo}

Introdução: A Osteoartrite (OA) é uma doença articular crônico-degenerativa que, quando sintomática, progride num padrão que inclui incapacidade para marcha e redução da Capacidade Funcional (CF), sendo sua manutenção um requisito importante para um envelhecimento saudável. Desse modo, vários recursos terapêuticos, entre eles a Fisioterapia Aquática (FA) e a Fisioterapia Terrestre (FT) surgem como alternativas para o tratamento dessa enfermidade. Objetivo: Avaliar o impacto da FA e da FT na CF de idosos com diagnóstico de OA de joelho. Materiais e métodos: Quinze voluntários com diagnóstico clínico e radiográfico de OA de joelho com idade entre 60 a 80 anos foram distribuídos aleatoriamente entre os grupos: FA ( $\mathrm{n}=5)$, que realizou cinesioterapia em imersão; FT ( $n=5$ ), que realizou cinesioterapia em solo; e grupo controle (GC) (n = 5), que não recebeu qualquer tipo de intervenção fisioterapêutica. A CF foi avaliada com base na velocidade da marcha usual, por meio de células fotoelétricas, nos movimentos de caminhar e subir e descer escadas. Resultados: Observou-se que os participantes da FA apresentaram melhora significativa no tempo da marcha usual $(p=0,007)$, marcha rápida $(p=0,02)$, subir escadas $(p=0,02)$ e descer escadas $(p=0,01)$, 
houve resultado satisfatório na FT apenas para descer escadas ( $p=0,04)$. Não foram encontradas diferenças significativas no GC. Conclusão: Por meio dos resultados obtidos neste estudo, conclui-se que a FA seja a mais indicada para tratamento da $\mathrm{OA}$, com resultados significativos na melhora da CF.

Palavras-chave: Osteoartrite. Idoso. Marcha. Modalidades de Fisioterapia.

\begin{abstract}
Introduction: Osteoarthritis (OA) is a chronic, degenerative joint disease that when it is symptomatic, it develops in a pattern that includes inability to walk and reduction of functional capacity ( $F C$ ), so its maintenance is an important requirement for a healthy aging. Thus, the aquatic physical therapy (AP) and the land one (LP) appear as alternatives for the treatment of this disease. Objective: To evaluate the impact of AP and LP on FC of elderly diagnosed with knee osteoarthritis. Materials and methods: Fifteen volunteers with clinical and radiographic diagnosis of knee $O A$ aged between 60 and 80 were randomly distributed among groups: $A P(n=5)$, that performed kinesiotherapy by immersion; $L P(n=5)$, that performed kinesiotherapy on soil; and the control group (CG) ( $n=5)$, that received no type of physical therapy intervention. The FC was evaluated for the time of usual gait speed, fast gait and going up and down the stairs, through photoelectric cells. Results: It was observed that the participants of AP showed significant improvement in the time of usual gait ( $p=0.07)$, fast gait $(p=0.02)$, going up $(p=0.02)$ and down the stairs $(p=0.01)$, There was satisfactory result in LP just for down the stairs ( $p=0.04)$. No significant differences were found in CG. Conclusion: Through the results of this study, to conclude that $A P$ is more suitable for the treatment of $O A$, with significantly results for improvement of $F C$.
\end{abstract}

Keywords: Osteoarthritis. Elderly. Gait. Modalitys of Physical therapy.

\section{Introdução}

O processo normal de envelhecimento se caracteriza pela diminuição da Capacidade Funcional (CF) dos diversos órgãos e tecidos, aumentando o risco de doenças crônico-degenerativas, na maior parte dos casos, que estão se tornando cada vez mais prevalentes em nosso país $(1,2,3)$.

Entre essas enfermidades podemos destacar a Osteoartrite (OA), uma doença articular crônico-degenerativa que evidencia alteração da cartilagem articular e do osso subcondral, levando a uma deformidade da articulação com a formação de osteófitos, os quais provocam dor, tumefação e rigidez articular na movimentação e refletem algum grau de sinovite e crepitação, indicativos de lesão cartilaginosa que evolui com o tempo $(4,5,6)$.

A prevalência da $\mathrm{OA}$ aumenta com a idade, ocorrendo mais frequentemente após os 60 anos. Aos 75 anos, 85\% das pessoas apresentam evidências radiológicas ou clínicas da doença, comprometendo os joelhos em $13,8 \%(7,8)$. Além do envelhecimento, obesidade, traumas, cirurgia articular, desequilíbrio hormonal, hereditariedade, nutrição e densidade óssea são alguns dos fatores que também predispõem à $\mathrm{OA}$ (4).
Os indivíduos com OA são, em sua maioria, assintomáticos. Quando sintomáticos, progridem em um padrão que inclui os seguintes sintomas: dor articular de duração e intensidade variáveis de acordo com o estado da doença; rigidez matinal de curta duração; crepitação óssea; disfunção física; edema; frouxidão dos ligamentos; diminuição ou perda do movimento; contraturas capsulares; fraqueza muscular; incapacidade para marcha; espasmo; fibrose; e alteração da propriocepção e equilíbrio $(5,6,9)$.

A incapacidade funcional pode ser definida como a inabilidade ou a dificuldade de realizar atividades de vida diária (AVD) que são indispensáveis para manter a independência e a participação na comunidade (10). Alterações funcionais objetivas também estão presentes em indivíduos com $\mathrm{OA}$, ocorrendo com frequência menor velocidade para marcha usual, marcha rápida e para subir ou descer escadas. Por essa razão, testes de velocidade são considerados métodos eficazes e simples, com baixos custos, para avaliar essas alterações funcionais (11).

Estudos da CF contribuem para avaliar o estado de saúde dos idosos, servindo como um importante indicador do processo saúde-doença $(8,12,13)$. 0 tratamento atual consiste, essencialmente, em combater 
a sintomatologia. Nesse sentido, a fisioterapia assume papel importante no que diz respeito à melhora dos sintomas e à restauração da função (10). A Fisioterapia Aquática (FA) é um recurso terapêutico da fisioterapia que atua como uma forma de cinesioterapia em imersão na água de piscinas. É um recurso bastante utilizado para tratamento de doenças reumáticas $(4,14-17)$. Outra opção amplamente utilizada para pacientes com OA é a Fisioterapia Terrestre (FT), modalidade da reabilitação que engloba os exercícios cinesioterapêuticos no solo em geral (18-22).

Desse modo, desenvolveu-se um estudo com o objetivo de analisar a eficácia da FA e da FT nos parâmetros da CF de indivíduos com OA de joelho - marcha usual, marcha rápida, subir e descer escadas - avaliados por meio de células fotoelétricas.

\section{Casuística e métodos}

Trata-se de um ensaio clínico com alocação sigilosa e aleatória dos participantes em três grupos. 0 projeto de pesquisa foi aprovado pelo Comitê de Ética do Hospital Regional de Assis sob o número 341/2010.

\section{Amostra}

A amostra deste estudo foi composta de idosos de ambos os gêneros, residentes no município de Assis (SP), com diagnóstico clínico e radiográfico de OA de joelho. Os idosos foram recrutados e encaminhados pela lista de espera do Setor de Fisioterapia Ortopédica de uma universidade do interior paulista, pelo ambulatório de especialidades da Secretaria Municipal da Saúde de Assis e por encaminhamentos de médicos aos pesquisadores responsáveis.

Todos os idosos selecionados para o estudo atenderam aos seguintes critérios de inclusão: idade entre 60 e 80 anos; diagnóstico de $0 \mathrm{~A}$ em um dos joelhos baseado nos critérios clínicos e radiográficos do American College of Rheumatology (22); não submissão a qualquer procedimento cirúrgico nos membros inferiores; não uso de qualquer auxílio à locomoção (bengalas, muletas, andadores); não submissão a tratamento de fisioterapia nos últimos três meses; não disfunção neurológica que promovesse alterações cognitivas; não contraindicação para hidroterapia como trombose venosa profunda, hiper ou hipotensão instável, radioterapia, diabetes mellitus instável, febre, doenças infecciosas, alterações sinais vitais, tímpanos perfurados, incontinência fecal e urinária, traqueostomia, cardiopatias, epilepsia.

Inicialmente, 96 indivíduos com OA foram contatados; destes, 15 foram considerados elegíveis por atenderem, rigorosamente, aos critérios de inclusão do estudo. Os 15 voluntários foram distribuídos de forma aleatória, por meio de sorteio realizado com uma urna, da qual os nomes dos participantes foram retirados um a um; o grupo FA foi constituído por cinco indivíduos com média de idade de 71,6 4 7,02 anos, o grupo FT foi formado por cinco indivíduos com idade média de 66,4 $\pm 5,12$ anos e o grupo controle (GC), constituído de cinco indivíduos com média de idade de 70,8 $\pm 6,30$ anos.

Antes do início da pesquisa, todos os voluntários receberam uma explicação detalhada sobre os objetivos e procedimentos da pesquisa, e assinaram o Termo de Consentimento Livre e Esclarecido, segundo as Diretrizes e Normas Regulamentadoras de Pesquisa envolvendo Seres Humanos constantes da Resolução do Conselho Nacional de Saúde n. 196/96.

\section{Avaliação da capacidade funcional}

A CF objetiva foi avaliada mediante a realização de quatro testes, a fim de verificar o tempo de: marcha usual, marcha rápida, subir escadas e descer escadas (11). Para a medição do tempo, foram utilizadas células fotoelétricas (Kit Multisprint ${ }^{\circledR}$, Inserra Indústria Mecânica Ltda, Belo Horizonte, Minas Gerais - Figuras 1 e 2). Esse equipamento permite a mensuração de testes de velocidade, agilidade e resistência, de eventos de longa ou curta duração, com maior fidedignidade dos resultados, pois sua precisão é de milissegundos.

Foram realizados testes de marcha em um corredor plano e sem obstáculos com distância de 25 metros. Os três primeiros e os dois últimos metros foram desprezados como aceleração e desaceleração. Para melhor compreensão do teste, os participantes percorreram o corredor uma vez.

Referente ao teste de marcha usual, os participantes foram orientados a "caminhar em um ritmo normal"; e para a marcha rápida, receberam a orientação de "caminhar o mais rápido possível", com o estímulo verbal "rápido, rápido" (11).

Os testes subir e descer escadas foram realizados em uma escada comum com corrimão. Para que os 


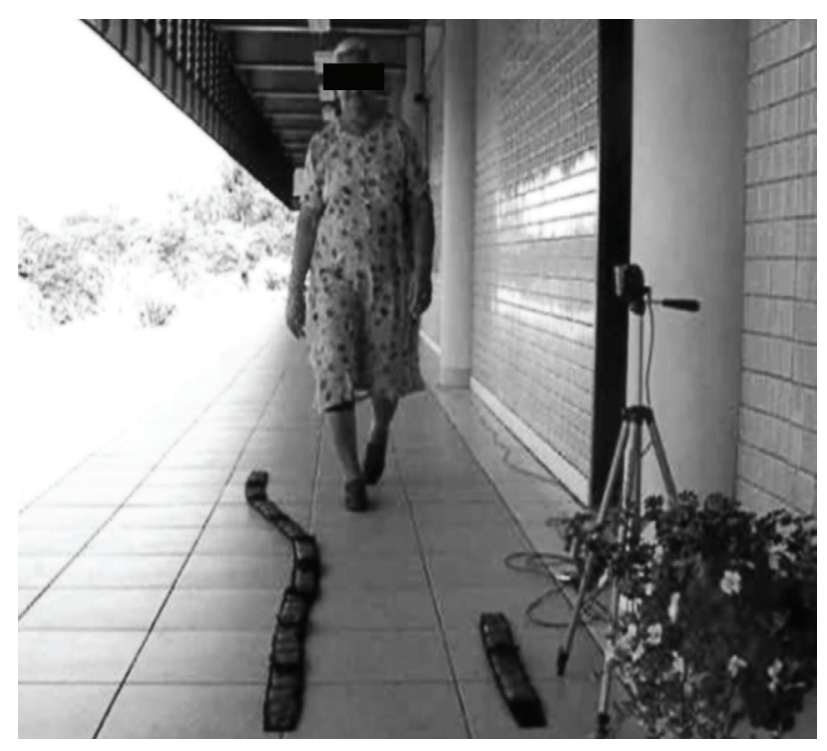

Figura 1 - Kit para Avaliação Computadorizada de Rendimento Físico MultiSprit

Fonte: Dados da pesquisa.

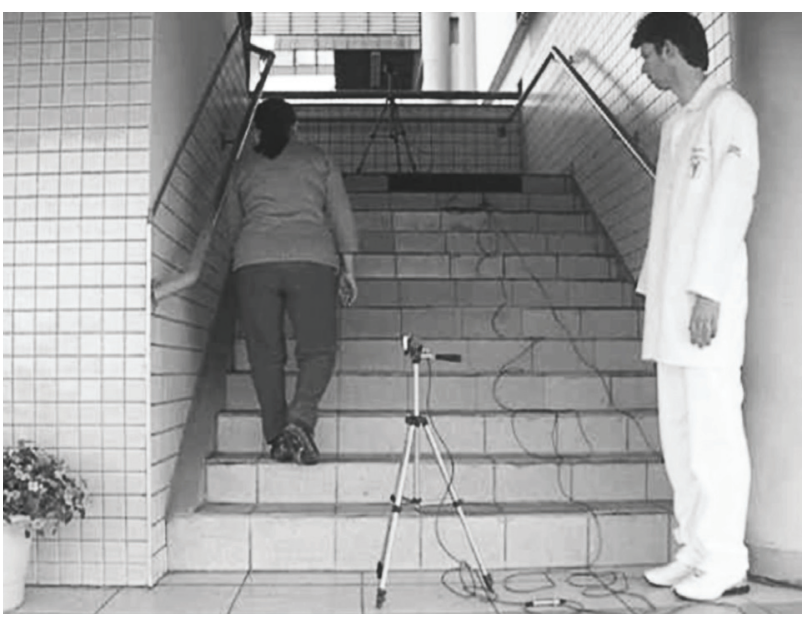

Figura 2 - Kit para Avaliação Computadorizada de Rendimento Físico MultiSprit

Fonte: Dados da pesquisa.

indivíduos se familiarizassem com o teste, todos subiram e desceram os 12 degraus uma vez. Todos foram instruídos a realizar a atividade "em um ritmo normal, da maneira como tivessem costume de fazer no dia a dia", sendo permitido o uso do corrimão. 0 mesmo padrão quanto ao uso do corrimão e ao tipo de apoio foram utilizados durante todo o teste (11).

Os participantes iniciaram os testes ao comando "já" do examinador. A contagem de tempo foi interrompida quando se alcançou o último degrau com os dois pés, sempre olhando para frente (11).
A ordem dos testes foi aleatorizada por sorteio e os participantes foram instruídos a utilizar um calçado usual, considerado por eles como confortável e seguro. Foram realizadas duas medidas para cada teste, com intervalo de 15 a 30 segundos entre cada medida e 30 a 60 segundos entre cada tipo de teste (11).

\section{Procedimento}

Previamente ao protocolo de tratamento, todos os participantes foram submetidos a uma avaliação para obtenção de dados demográficos, clínicos e antropométricos.

Os atendimentos da FA (Figuras 3 e 4) e FT (Figuras 5 e 6) foram realizados entre maio e setembro de 2010, três vezes por semana, totalizando 24 sessões com duração média de 60 minutos, com uma pausa de 45 dias entre a $12^{\underline{a}}$ e a $13^{\mathrm{a}}$ sessão.

As atividades da FA constituíam três etapas distintas: aquecimento, exercícios de fortalecimento muscular e resfriamento. A primeira etapa (aquecimento) envolvia exercícios de caminhada em velocidade progressiva seguidos de alongamento de cadeia muscular posterior e anterior dos membros inferiores. Na segunda etapa (cinesioterapia), realizaram-se exercícios progressivos de fortalecimento dos músculos dos membros inferiores: cadeia anterior, posterior, medial e lateral, treino de resistência do tríceps sural, exercícios em cadeia cinética fechada com uso de flutuador, "espaguete" e caminhada multidirecional. A progressão de tais exercícios pode ser observada no Quadro 1.

A terceira e última etapa (resfriamento) compreendeu uma caminhada leve pela piscina em velocidades regressivas. 0 paciente foi orientado a parar ao final da quinta volta (4).

Do mesmo modo, as atividades da FT foram compostas por uma série de exercícios progressivos. Os atendimentos foram realizados em quatro etapas distintas: analgesia, aquecimento, exercícios de fortalecimento muscular e resfriamento. Na primeira etapa foi realizada crioterapia sobre joelhos acometidos. Na segunda etapa (aquecimento) realizaram-se exercícios de caminhada em um circuito no solo, nas mesmas dimensões da piscina, em velocidade progressiva seguidos de alongamento de cadeia muscular posterior e anterior dos membros inferiores. A terceira etapa (cinesioterapia) também constituiu de exercícios de fortalecimento dos músculos 
dos membros inferiores: cadeia anterior, posterior, medial e lateral, treino de resistência do tríceps sural, exercícios em cadeia fechada e caminhada multidirecional. A progressão desses exercícios pode ser observada no Quadro 2.

A quarta etapa (resfriamento) consistiu de uma caminhada leve em velocidades regressivas. 0 paciente foi orientado a parar ao final da quinta volta.

As atividades dos grupos FA e FT tiveram uma progressão nos exercícios de treinamento muscular; assim, a cada sessão era incluído um novo exercício ao programa ou mudaram-se os números de séries e repetições; as atividades de aquecimento e resfriamento

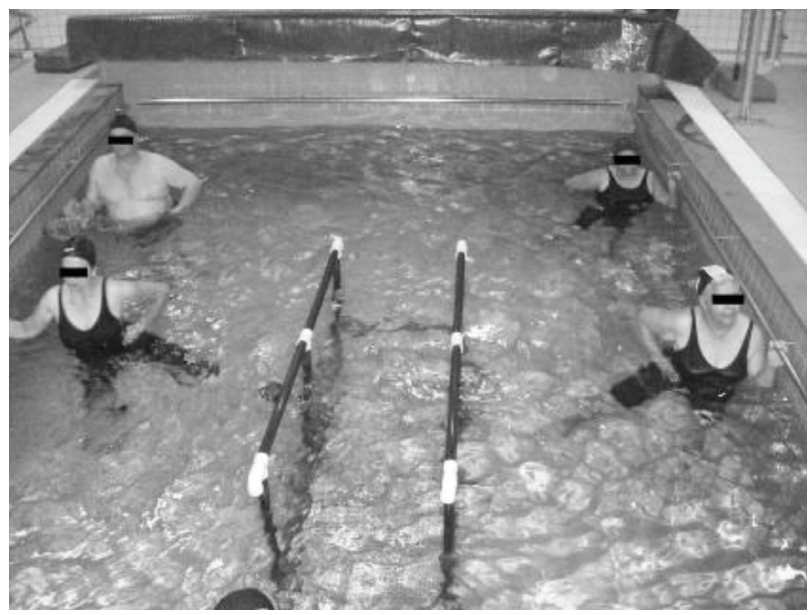

Figura 3 - Demonstração de exercícios de cadeia muscular lateral na FA

Fonte: Dados da pesquisa.

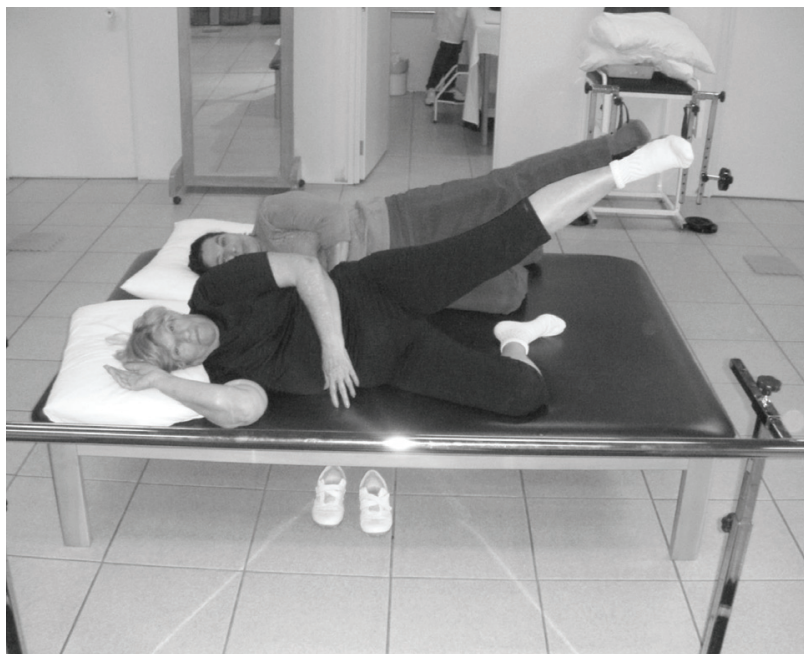

Figura 5 - Demonstração de exercícios de cadeia muscular lateral na FT

Fonte: Dados da pesquisa. estiveram presentes em todas as sessões. Da $6^{\underline{a}}$ à $12^{\underline{a}}$ sessão, o corpo das atividades já estava completo e a progressão dos exercícios foi individual, sendo exigido de cada participante o máximo de empenho na execução. Quanto à quantidade de séries executadas, no sétimo dia o número de séries passou a ser quatro e o número de repetições passou a ser 25 na $10^{a}$ sessão.

Após 12 sessões (43 dias após o início das intervenções) na FA e FT, realizou-se a segunda avaliação dos dados da CF, a fim de verificar a eficácia de cada intervenção. Após pausa de 45 dias entre a $12^{\underline{a}}$ e a 13 a sessão, realizou-se a terceira avaliação e, por fim, após 12 sessões, ocorreu a quarta e última avaliação.

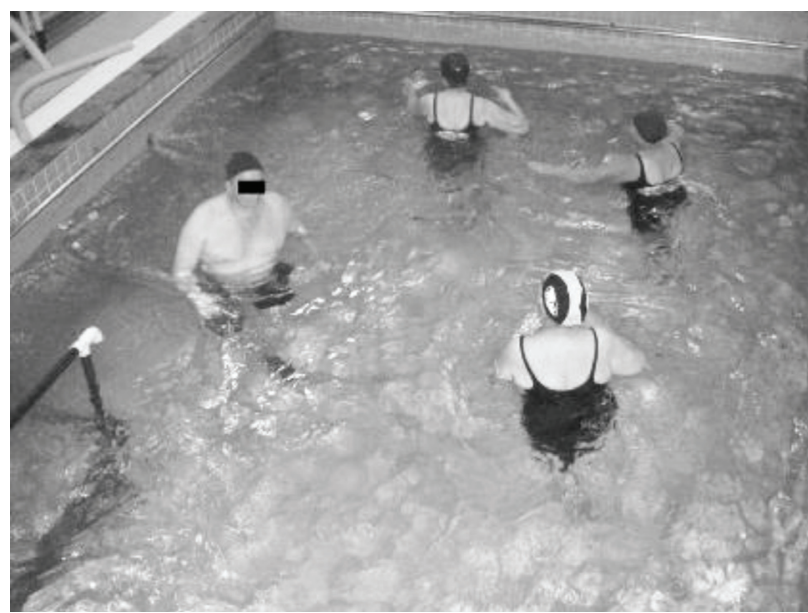

Figura 4 - Demonstração de caminhada multidirecional na FA

Fonte: Dados da pesquisa.

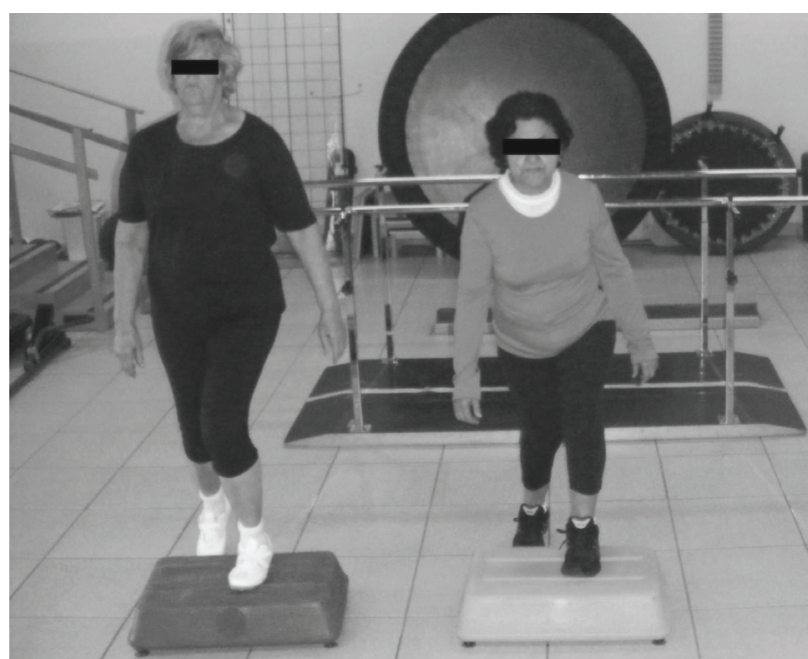

Figura 6 - Demonstração dos exercícios de cadeia cinética fechada (step) na FT

Fonte: Dados da pesquisa. 
Quadro 1 - Progressão do nível de dificuldade, séries e repetições dos exercícios de cinesioterapia em FA

\begin{tabular}{|c|c|c|c|}
\hline Etapa & Exercícios & Séries & Repetições \\
\hline $1^{\text {a }}$ & Adaptação & - & - \\
\hline $2^{\mathrm{a}}$ & $\begin{array}{l}\text { Exercícios da cadeia muscular anterior (flexão) } \\
\text { Exercícios da cadeia muscular posterior } \\
\text { (extensão) }\end{array}$ & 2 & 20 \\
\hline $3^{\mathrm{a}}$ & $\begin{array}{l}\text { Exercícios da } 2^{\mathrm{a}} \text { sessão acrescidos de cadeia } \\
\text { muscular lateral e medial (abdução e adução); }\end{array}$ & 2 & 20 \\
\hline $4^{\mathrm{a}}$ & $\begin{array}{l}\text { Exercícios da } 3^{\mathrm{a}} \text { sessão acrescidos de elevação } \\
\text { na ponta dos pés (plantiflexão) }\end{array}$ & 3 & 20 \\
\hline $5^{\mathrm{a}}$ & $\begin{array}{l}\text { Exercícios da } 4^{\mathrm{a}} \text { sessão acrescidos de exercício } \\
\text { de cadeia cinética fechada (flutuador) }\end{array}$ & 3 & 20 \\
\hline $6^{\mathrm{a}}$ & $\begin{array}{l}\text { Exercícios da } 5^{\mathrm{a}} \text { sessão acrescidos de caminhada } \\
\text { multidirecional }\end{array}$ & 4 & 20 \\
\hline $7^{\mathrm{a}}$ & Exercícios da $6^{\mathrm{a}}$ sessão & 4 & 20 \\
\hline $8^{\mathrm{a}}$ & Exercícios da $6^{0}$ sessão & 4 & 20 \\
\hline $9^{a}$ & Exercícios da $6^{0}$ sessão & 4 & 20 \\
\hline $10^{\mathrm{a}}$ & Exercícios da $6^{0}$ sessão & 4 & 20 \\
\hline $11^{\mathrm{a}}$ & Exercícios da $6^{0}$ sessão & 4 & 20 \\
\hline \multirow[t]{2}{*}{$12^{\mathrm{a}}$} & Exercícios da $6^{0}$ sessão & 4 & 20 \\
\hline & Pausa de 45 dias & - & - \\
\hline $13^{a}$ & Readaptação & - & - \\
\hline $14^{\mathrm{a}}$ & $\begin{array}{l}\text { Exercícios da cadeia muscular anterior (flexão) } \\
\text { Exercícios da cadeia muscular posterior } \\
\text { (extensão) }\end{array}$ & 2 & 20 \\
\hline $15^{\mathrm{a}}$ & $\begin{array}{l}\text { Exercícios da } 14^{0} \text { sessão acrescidos de cadeia } \\
\text { muscular lateral e medial (abdução e adução) }\end{array}$ & 2 & 20 \\
\hline $16^{\mathrm{a}}$ & $\begin{array}{l}\text { Exercícios da } 15^{\mathrm{a}} \text { sessão acrescidos de elevação } \\
\text { na ponta dos pés (plantiflexão) }\end{array}$ & 3 & 20 \\
\hline $17^{\mathrm{a}}$ & $\begin{array}{l}\text { Exercícios da } 16^{\text {a }} \text { sessão acrescidos de exercício } \\
\text { de cadeia cinética fechada (flutuador) }\end{array}$ & 3 & 20 \\
\hline $18^{\mathrm{a}}$ & $\begin{array}{l}\text { Exercícios da } 17^{\text {a }} \text { sessão acrescidos de } \\
\text { caminhada multidirecional }\end{array}$ & 3 & 20 \\
\hline $19^{a}$ & Exercícios da $18^{\mathrm{a}}$ sessão & 4 & 20 \\
\hline $20^{\mathrm{a}}$ & Exercícios da $18^{\mathrm{a}}$ sessão & 4 & 20 \\
\hline $21^{\mathrm{a}}$ & Exercícios da $18^{\mathrm{a}}$ sessão & 4 & 20 \\
\hline $22^{\mathrm{a}}$ & Exercícios da $18^{\mathrm{a}}$ sessão & 4 & 25 \\
\hline $23^{a}$ & Exercícios da $18^{\mathrm{a}}$ sessão & 4 & 25 \\
\hline $24^{\mathrm{a}}$ & Exercícios da $18^{\mathrm{a}}$ sessão & 4 & 25 \\
\hline
\end{tabular}

Fonte: Dados da pesquisa. 
Quadro 2 - Progressão do nível de dificuldade, séries e repetições dos exercícios de cinesioterapia da FT

\begin{tabular}{|c|c|c|c|}
\hline Etapa & Exercícios & Séries & Repetições \\
\hline $1^{\mathrm{a}}$ & Adaptação & - & - \\
\hline $2^{\mathrm{a}}$ & $\begin{array}{l}\text { Exercícios da cadeia muscular anterior (decúbito } \\
\text { dorsal com flexão de quadril) e exercícios da cadeia } \\
\text { muscular posterior (decúbito ventral com extensão } \\
\text { de quadril) }\end{array}$ & 2 & 20 \\
\hline $3^{\mathrm{a}}$ & $\begin{array}{l}\text { Exercícios da } 2^{\mathrm{a}} \text { sessão acrescidos de exercícios da } \\
\text { cadeia muscular lateral e medial (abdução e adução } \\
\text { de quadril contra a gravidade) }\end{array}$ & 2 & 20 \\
\hline $4^{\mathrm{a}}$ & $\begin{array}{l}\text { Exercícios da } 3^{\text {a }} \text { sessão acrescidos de exercícios de } \\
\text { elevação na ponta dos pés (plantiflexão) }\end{array}$ & 3 & 20 \\
\hline $5^{\mathrm{a}}$ & $\begin{array}{l}\text { Exercícios da } 4^{\mathrm{a}} \text { sessão acrescidos de exercício de } \\
\text { cadeia cinética fechada (step) }\end{array}$ & 3 & 20 \\
\hline $6^{\mathrm{a}}$ & $\begin{array}{l}\text { Exercícios da } 5^{\text {a }} \text { sessão acrescidos de caminhada } \\
\text { multidirecional }\end{array}$ & 3 & 20 \\
\hline $7^{\mathrm{a}}$ & Exercícios da $6^{a}$ sessão & 4 & 20 \\
\hline $8^{\mathrm{a}}$ & Exercícios da $6^{\mathrm{a}}$ sessão & 4 & 20 \\
\hline $9^{a}$ & Exercícios da $6^{\mathrm{a}}$ sessão & 4 & 20 \\
\hline $10^{\mathrm{a}}$ & Exercícios da $6^{\mathrm{a}}$ sessão & 4 & 25 \\
\hline $11^{\mathrm{a}}$ & Exercícios da $6^{\mathrm{a}}$ sessão & 4 & 25 \\
\hline \multirow[t]{2}{*}{$12^{\mathrm{a}}$} & Exercícios da $6^{\mathrm{a}}$ sessão & 4 & 25 \\
\hline & Pausa 45 dias & - & - \\
\hline $13^{\mathrm{a}}$ & Readaptação & - & - \\
\hline $14^{\mathrm{a}}$ & $\begin{array}{l}\text { Exercícios da cadeia muscular anterior (decúbito } \\
\text { dorsal com flexão de quadril) e exercícios da cadeia } \\
\text { muscular posterior (decúbito ventral e realizando } \\
\text { extensão de quadril) }\end{array}$ & 2 & 20 \\
\hline $15^{\mathrm{a}}$ & $\begin{array}{l}\text { Exercícios da } 14^{\mathrm{a}} \text { sessão acrescidos de exercícios } \\
\text { da cadeia muscular lateral e medial (abdução e } \\
\text { adução de quadril contra a gravidade) }\end{array}$ & 2 & 20 \\
\hline $16^{\mathrm{a}}$ & $\begin{array}{l}\text { Exercícios da } 15^{\mathrm{a}} \text { sessão acrescidos de exercícios } \\
\text { de elevação na ponta dos pés (plantiflexão) }\end{array}$ & 3 & 20 \\
\hline $17^{\mathrm{a}}$ & $\begin{array}{l}\text { Exercícios da } 16^{a} \text { sessão acrescidos de exercício de } \\
\text { cadeia cinética fechada (step) }\end{array}$ & 3 & 20 \\
\hline $18^{\mathrm{a}}$ & $\begin{array}{l}\text { Exercícios da } 17^{\text {a }} \text { sessão acrescidos de caminhada } \\
\text { multidirecional }\end{array}$ & 3 & 20 \\
\hline $19^{a}$ & Exercícios da $18^{\mathrm{a}}$ sessão & 4 & 20 \\
\hline $20^{\mathrm{a}}$ & Exercícios da $18^{\mathrm{a}}$ sessão & 4 & 20 \\
\hline $21^{\mathrm{a}}$ & Exercícios da $18^{\mathrm{a}}$ sessão & 4 & 20 \\
\hline $22^{\mathrm{a}}$ & Exercícios da $18^{\mathrm{a}}$ sessão & 4 & 25 \\
\hline $23^{\mathrm{a}}$ & Exercícios da $18^{\mathrm{a}}$ sessão & 4 & 25 \\
\hline $24^{\mathrm{a}}$ & Exercícios da $18^{\mathrm{a}}$ sessão & 4 & 25 \\
\hline
\end{tabular}

Fonte: Dados da pesquisa. 
Durante todo o período do atendimento, com o intuito de melhorar a qualidade na execução dos movimentos e a contagem de tempo dos exercícios (alongamentos) foram realizadas correções pelo pesquisador visando minimizar diferenças entre os pacientes.

Os participantes da FA foram constantemente encorajados a soltar o corrimão da piscina visando aumentar a exigência de controle muscular durante o exercício e também o treino de equilíbrio corporal.

Os participantes da GC não receberam qualquer tipo de intervenção fisioterapêutica durante os meses da pesquisa. Para assegurar a manutenção da condição desse grupo, ligações telefônicas semanais foram realizadas para cada participante, com o objetivo de acompanhar a não inclusão de qualquer intervenção terapêutica.

Ao término da aplicação do programa em FA e FT, o GC foi submetido a tratamento fisioterapêutico e, ao final da pesquisa, reuniram-se todos os participantes a fim de realizar uma apresentação expositiva sobre 0 A.

\section{Análise dos dados}

Para comparar as características observadas durante as avaliações, foram utilizadas as técnicas não paramétricas ANOVA de Friedman e, quando observada diferença significativa, a análise prosseguiu com o teste de Wilcoxon. 0 nível de significância utilizado para as conclusões das análises estatísticas foi de $5 \%$ $(\mathrm{p} \leq 0,05)$.

\section{Resultados}

Quatro participantes interromperam o acompanhamento, restando ao final da pesquisa três voluntários ( $\mathrm{n}=3)$ na FT, quatro $(\mathrm{n}=4)$ na FA e quatro $(n=4)$ no GC. As perdas ocorreram pelo excesso de faltas permitidas, por motivo de viagem e por condições clínicas não relacionadas à atividade (aumento da pressão arterial sistêmica).

A maioria dos participantes (60\%) apresentava acometimento bilateral, ou seja, $\mathrm{OA}$ em ambos os joelhos. Nove $(60 \%)$ referiram dor no joelho direito, quatro $(26,6 \%)$ no joelho esquerdo e apenas dois $(13,3 \%)$ referiram que ambas as articulações incomodavam na mesma intensidade.

Em relação à comparação entre os grupos nas variáveis $\mathrm{CF}$, foi observado que os participantes da FA apresentaram melhora significativa para o tempo da marcha usual ( $p=0,007)$ e da marcha rápida $(p=0,02)$. Não foram encontradas diferenças significativas no FT e GC para o tempo da marcha usual e da marcha rápida, conforme se observa nas Tabelas 1 e 2 .

Do mesmo modo, verificou-se melhora significativa no que se refere às avaliações do tempo de subir escadas $(p=0,02)$ na FA (Tabela 3 ).

Como demonstrado na Tabela 4, houve melhora significativa tanto dos participantes do FA $(p=0,01)$ como do FT $(p=0,04)$ no que se refere ao tempo de descer escadas.

\section{Discussão}

Indivíduos com OA apresentam deficit funcionais, ocorrendo com mais frequência menor velocidade para marcha e para subir ou descer escadas. Portanto, testes para avaliar o tempo para marcha são considerados métodos eficazes, simples e de baixo custo para avaliar essas disfunções (11).

Tabela 1 - Média, desvio padrão e valor de p da marcha usual de idosos com osteoartrose (OA) de joelho submetidos a tratamento aquático (FA) ou terrestre (FT) e de idosos do grupo controle (GC)

\begin{tabular}{lccc}
\hline & FA & FT & GC \\
\hline Avaliação 1 & $29,37 \pm 6,92$ & $25,35 \pm 5,44$ & $21,10 \pm 1,78$ \\
Avaliação 2 & $22,72 \pm 4,00$ & $21,83 \pm 3,72$ & $21,54 \pm 1,57$ \\
Avaliação 3 & $24,93 \pm 4,31$ & $20,90 \pm 1,46$ & - \\
Avaliação 4 & $21,23 \pm 3,45$ & $20,79 \pm 1,91$ & - \\
\hline p & $0,007^{*}$ & 0,12 & 0,71 \\
\hline
\end{tabular}

Legenda: * $=p<0,05$.

Fonte: Dados da pesquisa. 
Tabela 2 - Média, desvio padrão e valor de p da marcha rápida de idosos com osteoartrose (OA) de joelho submetidos a tratamento aquático (FA) ou terrestre (FT) e de idosos do grupo controle (GC)

\begin{tabular}{lccc}
\hline & FA & FT & GC \\
\hline Avaliação 1 & $18,99 \pm 4,04$ & $19,75 \pm 3,32$ & $17,14 \pm 2,27$ \\
Avaliação 2 & $16,42 \pm 2,52$ & $17,37 \pm 2,53$ & $17,51 \pm 0,83$ \\
Avaliação 3 & $18,28 \pm 2,62$ & $16,79 \pm 1,37$ & - \\
Avaliação 4 & $16,37 \pm 2,64$ & $16,06 \pm 0,85$ & - \\
\hline p & $0,02^{*}$ & 0,17 & 0,90 \\
\hline
\end{tabular}

Legenda: ${ }^{*}=p<0,05$.

Fonte: Dados da pesquisa.

Tabela 3 - Média, desvio padrão e valor de p dos dados relacionados a subir escadas de idosos com osteoartrose (OA) de joelho submetidos a tratamento aquático (FA) ou terrestre (FT) e de idosos do grupo controle (GC)

\begin{tabular}{lccc}
\hline & FA & FT & GC \\
\hline Avaliação 1 & $12,29 \pm 3,2$ & $9,67 \pm 2,01$ & $10,36 \pm 1,81$ \\
Avaliação 2 & $8,14 \pm 1,83$ & $7,21 \pm 0,99$ & $11,51 \pm 1,80$ \\
Avaliação 3 & $11,41 \pm 2,60$ & $7,25 \pm 0,83$ & - \\
Avaliação 4 & $9,78 \pm 2,93$ & $6,93 \pm 0,34$ & - \\
\hline $\mathbf{p}$ & $0,02^{*}$ & 0,12 & 0,14 \\
\hline
\end{tabular}

Legenda: * $=p<0,05$.

Fonte: Dados da pesquisa.

Tabela 4 - Média, desvio padrão e valor de p dos dados relacionados a descer escadas de idosos com osteoartrose (OA) de joelho submetidos a tratamento aquático (FA), terrestre (FT) e grupo controle (GC)

\begin{tabular}{lcrc}
\hline & FA & FT & GC \\
\hline Avaliação 1 & $13,58 \pm 5,42$ & $10,04 \pm 1,18$ & $11,76 \pm 3,93$ \\
Avaliação 2 & $9,56 \pm 4,17$ & $7,21 \pm 0,61$ & $12,59 \pm 2,80$ \\
Avaliação 3 & $12,20 \pm 4,80$ & $7,83 \pm 1,00$ & - \\
Avaliação 4 & $10,46 \pm 4,20$ & $6,84 \pm 0,88$ & - \\
\hline p & $0,01^{*}$ & $0,04^{*}$ & 0,27 \\
\hline
\end{tabular}

Legenda: * $=p<0,05$.

Fonte: Dados da pesquisa.

Sabendo disso, o referido estudo teve por prioridade verificar qual intervenção fisioterapêutica (FA e/ou FT) seria mais eficiente na recuperação da CF de idosos com OA de joelhos.

Os resultados demonstraram uma redução significativa no tempo da marcha usual, marcha rápida e de subir e descer escadas na FA quando comparados ao apresentado em FT e GC. Esses resultados são compatíveis com as informações de Candeloro e Caromano (23), que também não encontraram alterações no GC e indicaram como opção de tratamento a FA, devido à vantagem da diminuição do impacto nas articulações comparado ao solo.

Por outro lado, Foley, Halbert, Hewitt e Crotty (24) realizaram um ensaio clínico com 105 indivíduos e, referente à força muscular, não observaram 
diferenças significativas entre os grupos FA, exercícios de ginásio e GC. Todavia, os autores salientam que a melhora da dor ainda reporta à segurança dos exercícios realizados na piscina, por ser uma atividade com menor risco de exacerbação do processo inflamatório articular.

Caromano e Candeloro (25) destacam, ainda, que a FA é um recurso que utiliza efeitos físicos, fisiológicos e cinesiológicos advindos da imersão do corpo em piscina aquecida como recurso auxiliar da reabilitação ou prevenção de alterações funcionais.

Para indivíduos em tratamento de OA de joelho, os principais benefícios da FA são: redução da dor; manutenção ou aumento na amplitude de movimento articular e força muscular; melhora da habilidade funcional de marcha; relaxamento e alívio do espasmo muscular, promovendo a redução da carga, da rigidez e do edema nas articulações $(4,26)$.

Verificou-se, também, que a frequência de tratamento estabelecido nesse estudo, ou seja, três sessões semanais, foi eficiente para a melhoria da CF da amostra estudada.

Segundo Røgind, Bibow-Nielsen, Jensen, Møller, Frimodt-Møller e Bliddal (27), frequência, duração e intensidade do programa de exercícios podem afetar os resultados clínicos. No entanto, essa relação não tem sido bem estudada em pessoas com OA de joelho.

Outra proposta desse estudo foi enfatizar o trabalho muscular, tanto na FA quanto na FT, como abordagem terapêutica na reabilitação da OA de joelho. Observou-se redução significativa no tempo de descer escadas no grupo da FT. Uma revisão sistemática da Cochrane Collaboration (28) comparou os efeitos de diferentes tipos de exercício na OA de joelho. Constatou-se que todos os tratamentos individuais, em grupo e exercícios realizados em casa produzem resultados semelhantes com melhora da função física.

Para Bennel, Hunt, Wrigley, Lim e Hinman (29), a reabilitação muscular, como parte do regime de tratamento global para a OA do joelho, melhoraria a integridade articular e a função, reduzindo os sintomas e, por conseguinte, protegendo contra a progressão da OA. Nessa perspectiva, Alves, Leimann, Vasconcelos, Carvalho, Vasconcelos, Fonseca et al. (10) sugerem que as doenças crônicas apresentam uma forte influência na CF do idoso. Relatam, ainda, que poucos pesquisadores se preocupam em investigar o efeito de uma doença crônica específica na CF.
A CF é uma das mais importantes repercussões promovidas pela intervenção terapêutica em idosos. Está diretamente relacionada à independência para a realização das tarefas do cotidiano e, com isso, pode contribuir para a melhora da qualidade de vida dessa população $(30,31)$.

A funcionalidade é essencial para que idosos realizem suas AVDs com independência. Nesse contexto, a marcha é a base para a independência funcional. Portanto, preservar a funcionalidade dos indivíduos idosos é uma das principais diretrizes dos tratamentos fisioterapêuticos.

\section{Conclusão}

0 presente estudo demonstrou que os idosos com $\mathrm{OA}$ de joelho submetidos a FA obtiveram resultados significativamente satisfatórios no que se refere à redução do tempo da marcha usual, marcha rápida e de subir e descer escadas. Também os idosos submetidos a FT apresentaram melhora significativa no tempo referente ao teste de descer escadas. Observou-se, ainda, que os idosos com $\mathrm{OA}$ de joelho inseridos no GC, ou seja, que não realizaram intervenção fisioterapêutica, não apresentaram melhora da CF em nenhum dos parâmetros avaliados. No entanto, incentiva-se a realização de novos estudos com amostras representativas e delineamento metodológico semelhante, a fim de verificar a abordagem terapêutica mais eficiente na CF de idosos com OA de joelho.

\section{Referências}

1. Netto MP. Gerontologia. In: Yuaso DR, Sguizzatto GT. Fisioterapia em pacientes idosos. São Paulo: Atheneu; 2005. p. 331-47. doi:10.1590/ S0102-311X2003000300009.

2. Caldas CP. Envelhecimento com dependência: responsabilidades e demandas da família. Cad Saúde Pública 2003;19(3):733-81.

3. Christensen U, Stovring N, Schultz-Larsen K, Schroll M, Avlund K. Functional ability at age 75: is there an impact of physical inactivity from middle age to early old age? Scand J Med Sports. 2006, 16(4):245-51. doi:10.1111/j.1600-0838.2005.00459.x. 
4. Gomes WF. Impacto de um programa estruturado de fisioterapia aquática em idosas com osteoartrite de joelho. [Dissertação]. Belo Horizonte: Universidade Federal de Minas Gerais; 2007.

5. Okumura FA, Reis FA, Belchior ACG, Carvalho PTC, Silva BAK, Perreira DM, et al. Avaliação dos sintomas e capacidade física em indivíduos com osteoartrose de joelho. Rev Terapia Manual. 2009,7(30):83-87.

6. Zacaron KAM, Dias JMD, Abreu NS, Dias RC. Nível de atividade física, dor e edema suas relações com a disfunção muscular do joelho de idosos com osteoartrite. Rev Bras Fisioter. 2006;10(3):279-84. doi:10.1590/ S1413-35552006000300005.

7. Fuller R. Osteoartrose. In: Greve JMD' A. Tratado de medicina de reabilitação. São Paulo: Roca, 2007. p. 888-97.

8. Seda H, Seda AC. Osteoartrite. In: Moreira C, Carvalho, MAP. Reumatologia diagnóstico e tratamento. Rio de Janeiro: Guanabara Koogan; 2001. p. 289-94.

9. Pereira LSM, Dias RC, Dias JMD, Gomes GC, Sitta MI. Fisioterapia. In: Freitas EV, Py L, Néri AL, Cançado FAX, Gorzoni ML, Rocha SM. Tratado de geriatria e gerontologia. Rio de Janeiro: Guanabara Koogan, 2002. p. 846-50.

10. Alves LC, Leimann BCQ Vasconcelos MEL, Carvalho MS, Vasconcelos AGG, Fonseca TCO, et al. A influência das doenças crônicas na capacidade funcional dos idosos do município de São Paulo, Brasil. Cad. Saúde Pública. 2007;23:1924-30. doi:10.1590/ S0102-311X2007000800019.

11. Vasconcelos KSS, Dias JMD, Dias RC. Relação entre intensidade de dor e capacidade funcional em indivíduos obesos com osteoartrite de joelho. Rev Bras Fisioter. 2006,10(2):213-18. doi:10.1590/ S1413-35552006000200012.

12. Nogueira S, Ribeiro RCL, Rosado LPEL, Franceschini SCC, Ribeiro AQ, Perreira AQ. Fatores determinantes da capacidade funcional em idosos longevos. Rev Bras Fisioter. 2010;14:322-29. doi:10.1590/ S1413-35552010005000019.

13. Nunes MC, Ribeiro RCL, Rosado EPFL, Franceschini SC. Influência das características sociodemográficas na capacidade funcional de idosos residentes em Ubá, Minas Gerais. Rev Bras Fisioter. 2009;13(5)376-82.
14. Templeton MS, Booth DL, O'Kelly WD. Effects of aquatic therapy on joint flexibility and functional ability in subjects with rheumatic disease. J Orthop Sports Phys Ther. 1996;23(6):376-81.

15. Stenstrom CH, Lindell B, Swanberg E, Swanberg P, Harns-Rindgahl K, Nordemar R. Intensive dynamic training in water for rheumatic arthritis functional class II - a long-term study of effects. Scand J Rheumatol. 1991;20(5):358-65.

16. Ruoti RG, Morris D, Cole AJ. Reabilitação aquática de pacientes com disfunções músculo-esqueleticas das extremidades. São Paulo: Manole; 2000. p. 67-93.

17. Caromano FA, Nowotny JP. Princípios físicos que fundamentam a hidroterapia. Rev Fisioter Bras. 2002; $3: 1-9$

18. Biasoli MC, Izola LNT. Aspectos gerais da reabilitação física em pacientes com osteoartrose. Rev Bras Med. 2003;60:133-6.

19. French HP, Cusack T, Brennan A, White B, Gilsenan C, Fitzpatrick $\mathrm{M}$, et al. Exercise and manual physiotherapy arthritis research trial (EMPART): a multicentre randomized controlled trial. BMC Musculoskeletal Disorders. 2009;10:9. doi:10.1186/1471-2474-10-9.

20. Groot JB, Bussmann JB, Stam HJ, Verhaar JAN. Actual everyday physical activity in patients with end-stage hip or knee osteoarthritis compared with healthy controls. Osteoarthr Cartilage. 2008;16:436-42. doi:10.1016/j.joca.2007.08.010.

21. Lange AK, Vanwanseele B, Fiatarone Singh MA. Strength training for treatment of osteoarthritis of the knee: a systematic review. Arthritis Rheum. 2008;59(10):1488-94. doi:10.1002/art.24118.

22. Tamegushi AS, Trelha CS, Dellaroza MSG, Cabrera M, Ribeiro TN. Capacidade funcional de idosos com osteoartrite de joelhos e quadril. Rev Espa P. Sau. 2008; $9(2): 8-16$.

23. Candeloro JM, Caromano FA. Efeito de um programa de hidroterapia na flexibilidade e na força muscular de idosas. Rev Bras Fisioter. 2007;11(4):303-9.

24. Foley A, Halbert J, Hewitt T, Crotty M. Does hydrotherapy improve strength and physical function in patients with osteoarthritis - a randomized controlled trial comparing a gym based and hydrotherapy based strengthening programme. Ann Rheum Dis. 2003; 62:1162-67. doi:10.1136/ard.2002.005272. 
25. Caromano FA, Candeloro JM. Fundamentos da hidroterapia para idosos. Arq. Ciências Saúde Unipar. 2001;5:187-95.

26. Ishik CYW. Fisioterapia aquática em reumatologia. In: Sacchelli T, Accacio LMP, Radl ALM. Fisioterapia aquática: Barueri: Manole; 2007. p. 241-58.

27. Røgind H, Bibow-Nielsen B, Jensen B, Møller HC, Frimodt-Møller $\mathrm{H}$, Bliddal $\mathrm{H}$. The effects of a physical training program on patients with osteoarthritis of the knees. Arch Phys Med Rehabil. 1998,79:1421-27. doi:10.1016/S0003-9993(98)90238-6.

28. Fransen M, McConnell S, Bell M. Exercise for osteoarthritis of the hip or knee Cochrane Database Syst Rev. 2003;3.

29. Bennell KL, Hunt MA, Wrigley TV, Lim BW, Hinman RS. Muscle and exercise in the prevention and management of knee osteo arthritis: an internal medicine specialist's guide. 2009;93(1):161-77.
30. Pereira LSM, Dias RC, Dias JMD, Gomes GC, Sitta MI. Fisioterapia. In: Freitas EV, Py L, Neri AL, Cansado FLX, Gorzoni ML, Rocha SM. Tratado de geriatria e gerontologia. Rio de Janeiro: Guanabara Koogan; 2002. p. 46-56.

31. Cardoso JH, Costa JSDC. Características epidemiológicas, capacidade funcional e fatores associados em idosos de um plano de saúde. Ci Saúde Col. 2010, 15:287178. doi:10.1590/S1413-81232010000600024.

Recebido: 19/05/2012

Received:05/19/2012

Aprovado: 09/01/2013

Approved: 01/09/2013 